\title{
ACADEMIA $\mid$ Letters \\ Salutogenesis in the time of COVID-19: What coping resources enable people to face the crisis and stay well? International and longitudinal study
}

\author{
Adi Mana, Peres Academic Center, Israel \\ Rute Grossi-Milani, Universidade Cesumar (UNICESUMAR), Maringá, Paraná, \\ Brazil \\ Fabiane Dolphine Fuentes Penachiotti, Universidade Cesumar (UNICESUMAR), \\ Maringá, Paraná, Brazil \\ Lisa. J. Hardy, Northern Arizona University, U.S.A \\ Dolors Juvinyà Canal, University of Girona, Spain \\ Sharon Benheim, Ben Gurion University of the Negev, Israel \\ Roald Pijpker, Wageningen University, The Netherlands \\ Kristal Polhuis, Wageningen University, The Netherlands \\ Moran Neuman, Ben Gurion University of the Negev, Israel \\ Melody Hakimian, Northern Arizona University, U.S.A \\ Shifra Sagy, Ben Gurion University of the Negev, Israel
}

\begin{abstract}
Scientific Background: Understanding, responding to, and preventing disasters requires a multidisciplinary approach. During COVID-19 there have been contributions by bench scientists studying the pathogenic aspects of the illness as well as medical and social scientists understanding the multi-layered impacts of the global pandemic. Our study is based on the salutogenic approach which asks about the individual and collective perceptions of coping


resources available and the relationship between these resources and levels of anxiety and mental health. We also studied the role of the socio-national context in which the crisis occurred and whether it may also contribute to the understanding of the reactions of individuals in the unusual event of the COVID-19 crisis. Objectives: The study examined the personal (sense of coherence), social resources (social support), and national resources (trust in public institutions and leaders, and sense of national coherence), that enabled high levels of mental health and low levels of anxiety in time of the pandemic crisis. Methodology: Our international study was carried out in nine countries: Israel, Italy, Spain, the Netherlands, Germany, Switzerland, Austria, Brazil, the U.S.A, during the time of the COVID-19 pandemic outbreak. We conducted a longitudinal study in Israel during six phases during the first year of the crisis. Findings: We found that Sense of coherence (SOC) was a main and stable coping resource among the participants from all countries included in the study. However, differences were found in the levels of perceptions of national coping resources among different social groups. Conclusions: A strong SOC is crucial for health and survival during times of global and local crises. Policy Implications/recommendations: During regular times, and especially in times of crisis, leaders, and policymakers should prioritize strengthening the sense of coherence of the population. Therefore, the messages to the public should be created and designed to enhance comprehensibility, manageability, and meaningfulness of the pandemic and trust in government and public health initiatives.

\section{Scientific Background}

The COVID-19 pandemic continues to affect the lives of millions worldwide, causing great uncertainty and anxiety. In addition to severe physical health consequences, the pandemic is also having an impact on the general population in terms of mental health and well-being. Differences in the responses to the pandemic were observed between and within countries, as individuals and nations differ in resources, political, economic, and social factors that they drew on to cope with such a crisis. Our study employs the salutogenic approach (Antonovsky, 1987) that, in contrast to pathogenesis, focuses on the study of the origins of health, illuminates salutary factors that promote health, reduce distress reactions, and explain successful coping with stress. The study examined the personal (sense of coherence), social resources (social support), and national resources (trust in public institutions and leaders who were responsible for managing the COVID-19 crisis and sense of national coherence), that enabled high levels of mental health and low levels of anxiety in time of the pandemic crisis. 


\section{Objectives}

The aims of our study were (1) to study which resources play a role in coping with a pandemic in a mental health-promoting way, (2) to compare the pattern of these resources in promoting mental health and reducing anxiety levels across countries, and (3) evaluate the longitudinal effects of the crisis on mental health and anxiety, while moving from an acute to a chronic stress situation during the COVID-19 crisis. Those aims were achieved by an international study comparing nine countries: Israel, the Netherlands, Italy, Spain, Germany, Switzerland, Austria, Brazil, and the U.S.A, during the outbreak of COVID-19 pandemic, and by a longitudinal study conducted in Israel in six phases throughout the first year of the crisis.

\section{Methodology}

\section{The research variables}

The dependent variables: We explored mental health using the MHC-SF questionnaire which tests emotional, psychological, and social well-being. General anxiety was explored by GAD7 questionnaire as a pathogenic outcome of stressful situations as it is usually considered the main result of situations of stress.

\section{The independent variables}

Coping resources: Coping resources were explored on the individual, social, and national levels: At the individual level, we explored the sense of coherence (SOC, Antonovsky 1987) the core coping resource in the salutogenic model- which is an underlying resource enabling people to choose successful coping strategies. SOC expresses an individual view of the world as comprehensible (the extent to which stimuli from one's external and internal environment are perceived as structured, explicable, and predictable), manageable (the extent to which resources are perceived as available to a person to meet the demands posed by these stimuli), and meaningful (the extent to which these demands are perceived as challenges, worthy of investment, and engagement) (Antonovsky, 1987). At the social level, we explored the coping resource of perceived social support (from family, friends, colleagues, and virtual networks), a subjective feeling of being supported and cared for by others, and having a reliable network to turn to when needed, in everyday situations or in specific moments of crisis. Coping resources on the national level were a sense of national coherence (SONC) and trust in formal institutions. SONC is based on Antonovsky's components of SOC and is defined as an enduring tendency to perceive one's national group as comprehensible, meaningful, and manageable, 
while trust in institutions has been assessed in several studies as a coping resource that could help people to feel safer and more confident. In addition, we examined situational factors self-reported perceptions of one's level of health and economic risks due to the COVID-19 crisis, and belonging to social groups: gender, age groups, marital status, religiosity, and belonging to right-wing or left-wing political groupings. Participants: Data for the international study was collected via online survey platform and social media networks and included participants (age 18-93) from Israel ( $\mathrm{n}=669)$, Italy $(\mathrm{n}=899)$, Spain $(\mathrm{n}=476)$, the Netherlands $(\mathrm{N}=622)$, Germany $(\mathrm{n}=708)$, Austria $(\mathrm{n}=1026)$, Switzerland $(\mathrm{n}=147)$, the U.S.A $(\mathrm{n}=1200)$ and Brazil $(n=2402)$. The longitudinal study in Israel included about 600 participants whose data were collected in six phases along with the Covid-19 ways in the first year (from March 2020 until September 2021).

\section{Main findings of the international study}

In all the samples, SOC was the main predictor of mental health and reduced level of general anxiety, however, different patterns of coping resources and risk factors were related to the salutogenic reaction (mental health) and the pathogenic reactions (general anxiety). The perception of economic risk due to the pandemic was a better predictor of anxiety and mental health as compared to the perception of the health risk (Mana et. al., 2021ab). Perceived social support mediated the relationships between SOC and mental health (Mana et al., 2021b). For participants with political orientations in opposition to their government, the political threat was stronger than the health crisis and they were reported on lower levels of mental health (Mana \& Sagy, 2020; Hardy et al., 2021).

\section{Main findings of the longitudinal study in Israel}

Mental health levels decreased along with the research phases, while anxiety levels were higher at the outbreak of the pandemic as compared to the later phases. SOC levels remained stable while the other coping resources levels gradually decreased. Strong correlations were found between the level of SOC at the outbreak of the pandemic and the mental health level after a year of COVID-19 crisis (Mana et al., 2021c). 


\section{Conclusions}

Examining the coping process during the COVID-19 pandemic that has shaken our world, has given us insights into the global and local dynamic process of coping with an acute stressful situation that developed into a chronic one. Each type of stress situation demands quite different resources, but our findings indicate that in a process in which an acute or chronic threat is experienced, the ability to perceive the world as comprehensible, manageable, and meaningful, is the most important resource enabling people to identify and use effective coping strategies.

\section{Policy Implications/recommendations}

Understanding the role of coping resources in promoting mental health during a crisis, especially SOC, can lead to a more holistic and salutogenic approach by the health care system. Based on our findings of the central role of SOC in predicting the mental health of the global population, we suggest exploring SOC in ongoing international public health and social surveys. Moreover, when leaders, health workers, and governmental and local authorities address the public in time of crisis, more effort should be invested in crafting messages that help people's perception of the world or their collective as more comprehensive, manageable, and meaningful. 


\section{References}

Antonovsky A. (1987). Unraveling the mystery of health. How people manage stress and stay well. Jossey-Bass.

Hardy, L.J., Mana, A., Mundell, L., Neuman, M., Benheim, S., \& Otenyo, E. (2021). Who is to blame for COVID-19? Examining politicized fear and health behavior through a mixed methods study in the United States. PLoS ONE, 16(9): e0256136. https://doi.org/10.1371/ journal.pone. 0256136

Mana, A., Bauer, A., Meier Magistretti, C., Sardu, C., Juvinya Canal, D., Hardy, L.J., Catz, O., Tušl, M., \& Sagy, S. (2021b). Order out of chaos: Sense of coherence and the mediating role of coping resources in predicting mental health during COVID-19 in 7 countries. Social Science \& Mental Health (SSM - Mental Health), 1, 100001. https://doi.org/10. 1016/j.ssmmh.2021.100001

Mana, A., Catz, O., Mana, Y., Neuman, M., Benheim, S., \& Sagy. S. (2021c). How do people cope during the COVID-19 pandemic and stay well? A salutogenic longitudinal study in Israel. Frontiers in psychology, 12, 4377. https://www.frontiersin.org/article/10. 3389/fpsyg.2021.729543

Mana, A., Super, S., Sardu, C., Juvinya Canal, D., Neuman, M., \& Sagy, S. (2021a). Individual, social and national coping resources and their relationships with mental health and anxiety: A comparative study in Israel, Italy, Spain, and the Netherlands during the Coronavirus pandemic. Global Health Promotion. 18, https://doi.org/10.1177/1757975921992957

Mana, A., \& Sagy, S. )2020(. Brief report: Can political orientation explain mental health in the time of a global pandemic? Voting patterns, personal and national coping resources, and mental health during the Coronavirus crisis, Journal of Social and Clinical Psychology, 39(3), 187-193. https://doi.org/10.1521/jscp.2020.39.3.165 